\title{
EFFECTS OF STIMULATIONS OF THE PYRAMIDAL TRACT AND STRIATE BODY UPON SPINAL MOTONEURONS
}

\author{
Kazuo Sasaki, Akira Namikawa and Morio Matsunaga* \\ Department of Physiology and 1 st Surgical Division, \\ Faculty of Medicine, Kyoto University, Kyoto
}

\begin{abstract}
Studies on the influence of the upper brain centers upon the spinal activity have become very numerous. Some centers are considered to be facilitatory while others are inhibitory, all contributing to the integrative action of the central nervous system. However, as to the mechanisms underlying those facilitatory or inhibitory action, only a few investigations have so far been reported. One of the most recent papers along this line is that of KoIZUMI, UshiYAMA and BRoOKs ${ }^{4}$, which described the reticular formation action on spinal interneurons and motoneurons. In their work, changes occurring in the membrane potential of individual units were studied.

Recently we have been engaged in the study of influence exerted by upper brain centers upon the spinal motoneurons with the aid of intracellular potential recording. The first part of these studies concerning midbrain centers has already been reported ${ }^{10)}$. In the work to be reported here, changes in individual motoneurons upon stimulating pyramidal decussation, internal capsule and the striate body were studied.
\end{abstract}

\section{METHODS}

Over thirty adult cats were used for this study. Experimental procedures were for the most part similar to those of our previous study ${ }^{10)}$. Laminectomy of lumbosacral segments and craniotomy were performed under Nembutal anesthesia $(25 \mathrm{mg} / \mathrm{kg}$., intraperitoneal injection), and bilateral peroneal and tibial nerves were exposed with peripheral ends sectioned. The animal was then mounted on a fixing apparatus which consisted of two parts. The anterior part is a stereotaxic instrument of Horsley-Clarke type used for head and the posterior part is a fixing apparatus for the lumbosacral cord. The exposed part of spinal cord was protected with warmed liquid parafine. The animal was then left untouched

Received for publication December 15, 1959.

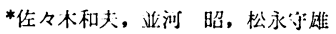


for several hours without any additional administration of anesthetics.

The electrodes used for stimulation of the upper foci were made of steel wires, about $0.3 \mathrm{~mm}$. in diameter and coated except the tip. For stimulation of striate body and internal capsule, three to five pairs of these electrodes were bundled up in advance and fixed to a manipulator with the relative position of tips so arranged that, when they were inserted en bloc into the brain to a certain depth, the tips of each pair were located in the focus to be stimulated. The relative position of the tips was determined referring to the stereotaxic atlas by JASPER. The pyramidal decussation was approached from the dorsum of medulla oblongata. Three pairs of steel wire electrodes, which were fixed in parallel with, and $2 \mathrm{~mm}$. apart from each other, were inserted ventro-rostrally from the bulbar dorsum, so that the tips of the electrode pairs were located in the pyramidal decussation in the midline and in two loci in the bulbar reticular formation, $2 \mathrm{~mm}$. and $4 \mathrm{~mm}$. lateral to the midline respectively. The latter two pairs of electrodes were used for control experiment in order to assure the specificity of the results obtained by pyramidal decussation stimulation. A brief series lasting 2-3 msec. of rectangular current pulses (pulse width: $0.05-0.10 \mathrm{msec}$; frequency: 5,000-10,000/sec.; voltage: 3-6 V) was given to each focus to be stimulated.

Single rectangular current pulses of $0.3 \mathrm{msec}$. duration were applied as stimuli to dorsal roots and peroneal and tibial nerves. After sectioning the ventral roots $\mathrm{L}_{6}$ and $\mathrm{L}_{7}$ bilaterally, the sectioned roots of the same side were put together and placed on a pair of silver electrodes, one of which was situated close to the spinal cord and the other at the severed ends of the roots, in order to pick up ventral root electrotonic potentials. A RC coupled amplifier (time constant: $0.5 \mathrm{sec}$.) feeding a cathode-ray oscilloscope was used for recording from the ventral roots as well as from the peripheral nerves.

For recording intracellular potentials from the spinal motoneurons glass micropipettes were used, whose resistance was $10-20 \mathrm{M} \Omega$ when filled with $\mathrm{M}-\mathrm{KCl}$. The micropipettes were inserted from the dorsal surface of the cord at the level of $\mathrm{L}_{6}-\mathrm{L}_{7}$, with the aid of a micromanipulator of Peterfi type. A DC amplifier with cathode-following input was used for intracellular recording.

Histological checking. At the end of experiment, anodic currents were passed through the stimulating electrodes in the brain and iron ions which flowed out into the brain tissue were stained histochemically with the method of TIRMANNSchmelzer. The location of the electrode tips was thus examined by making serial sections after each experiment.

RESULTS

(1) Ventral root potentials induced by stimulating pyramidal decussation, internal capsule and striate body.

The ventral root potentials induced by stimulating upper brain structures 
were recorded from $L_{6}$ and $L_{7}$ ventral roots of both sides. The potentials included both spike discharges and the electrotonic potentials, since the proximal lead was situated as close as possible to the spinal cord. Upon stimulating the pyramidal decussation, intense initial spikes were discharged, followed by a slow negative deflection which was most likely to be electrotonic in nature (FIG. 1, PD). Records obtained from the peroneal and tibial nerves before sectioning the ventral roots showed corresponding efferent outflows of approximately same size in both nerves. Since the stimulating electrodes were located rostro-caudally in the midline, the effects were almost alike on both sides.

The stimulation of the internal capsule brought about more complicated potentials in the ventral roots of the opposite side. They were often initiated by a slow positive deflection which was followed by spike discharges and then by irregular negative deflections (FIG. 1 IC).

The stimulation of the striate body induced slow positive deflection which was especially conspicuous in the case of globus pallidus (FIG. 1 GP). The caudate nucleus produced a similar positive deflection less conspicuous and with a little longer latent time than globus pallidus.

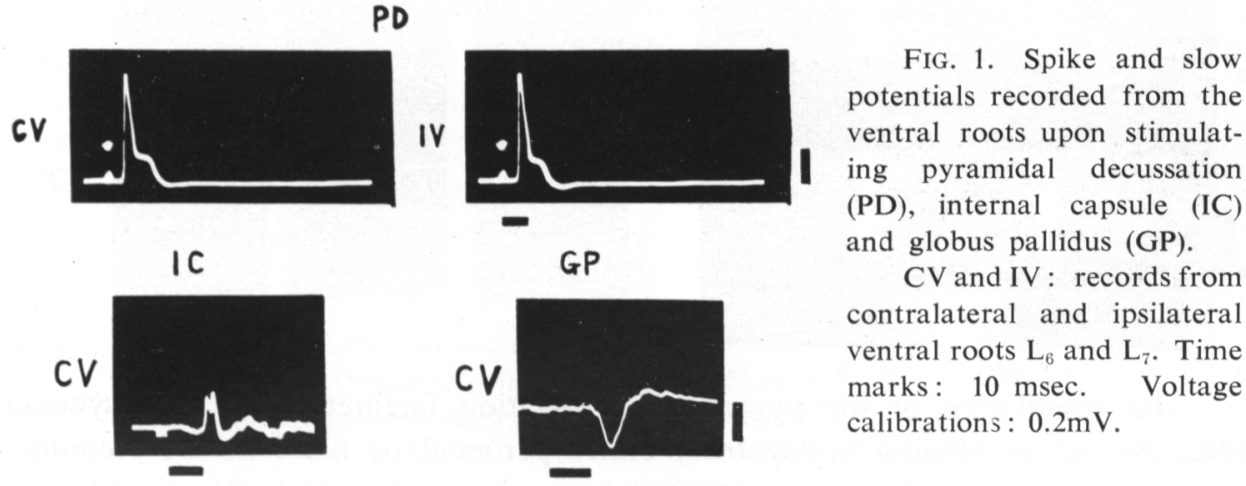

(II) Influences upon the spinal monosynaptic reflex.

The intensity of stimuli to the pyramidal decussation was then reduced to a subthreshold value for inducing spinal outflow and the stimuli were given associated with peripheral nerve stimulation at varying time intervals, in order to study the influence of pyramidal stimulation upon the spinal monosynaptic reflex. The peripheral nerve stimulated was the peroneal or tibial nerve and the stimulus intensity was submaximal to group Ia fibers in each nerve. Monosynaptic reflex discharges in the ventral roots which were thus evoked at various moments before and after conditioning, were recorded superimposed on a single frame, as shown half-schematically in FIG. 2. By a similar way, effects of stimulating striate body on the monosynaptic reflexes were also investigated. 


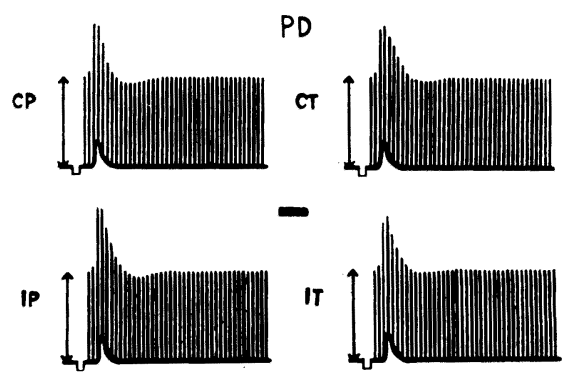

GP

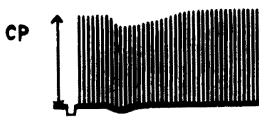

CT

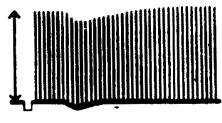

IP

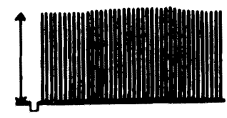

IT

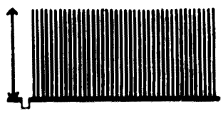

$\mathrm{CN}$

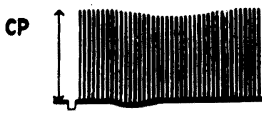

cr

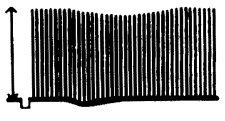

FIG. 2. Facilitation and inhibition of the monosynaptic spinal reflex resulted from stimulations of pyramidal deccussation, globus pallidus and caudate nucleus. Half-schematic representation.

At various times after stimulation of upper foci (shown by a rectangular depression near the left end of the base-line), a test stimulus was applied to peripheral nerves (CT, IT, CP and IP: contralateral and ipsilateral, tibial and peroneal nerves). Vertical lines indicate spike sizes in the ventral roots $\mathrm{L}_{6}$ and $\mathrm{L}_{i}$. Vertical arrows on the left end indicate control reflex size. Undulations of the base-line show ventral root slow potentials elicited by stimulating respective upper focus. Horizontal bars: $10 \mathrm{msec}$.

IP

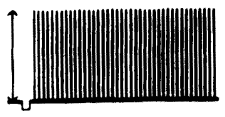

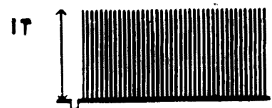

The stimulation of the pyramidal decussation facilitated the monosynaptic reflex due to an afferent volley from either peroneal or tibial nerve, seemingly to the same extent. The range of time intervals within which the conditioning was effective seemed to coincide with the duration of the negative ventral root potential. On the average, this range of facilitation was somewhat broader for the tibial monosynaptic reflex than for the peroneal. Needless to say, the facilitation was observed bilaterally.

As shown in Fig. 2, globus pallidus exerted an inhibition upon the monosynaptic reflex induced from both peroneal and tibial nerves of the contralateral side. The inhibition corresponded obviously to the positive ventral root potential which was observed only contralaterally in response to the global stimulation. No influence was observed on the monosynaptic reflex of the ipsilateral side.

Similar inhibition of the contralateral monosynaptic reflex induced by peroneal as well as tibial nerve stimulation was observed by stimulating the caudate nucleus. The inhibition was less pronounced than that induced from the globus 
pallidus, being initiated with a longer latency and lasting a little longer. No influence was observed on the monosynaptic reflex of the ipsilateral side.

The stimulation of the putamen resulted sometimes in facilitation and other times in inhibition or in a complicated effect comprising inhibition and facilitation. (III) Effects on the intracellular potential of lumbar alpha motoneurons.

In the spinal cord connected with intact brain under slightest degree of anesthesia, some motoneurons were firing repetitively, while others were silent, as has been reported in the previous paper ${ }^{10)}$. Figs. 3 and 4 give the records of the intracellular potentials of three flexor and three extensor motoneurons when the upper foci of the contralateral side were stimulated. The motoneurons innervating the flexors or extensors were identified by the presence of the monosynaptic reflex discharges in response to a peroneal or tibial nerve stimulation.

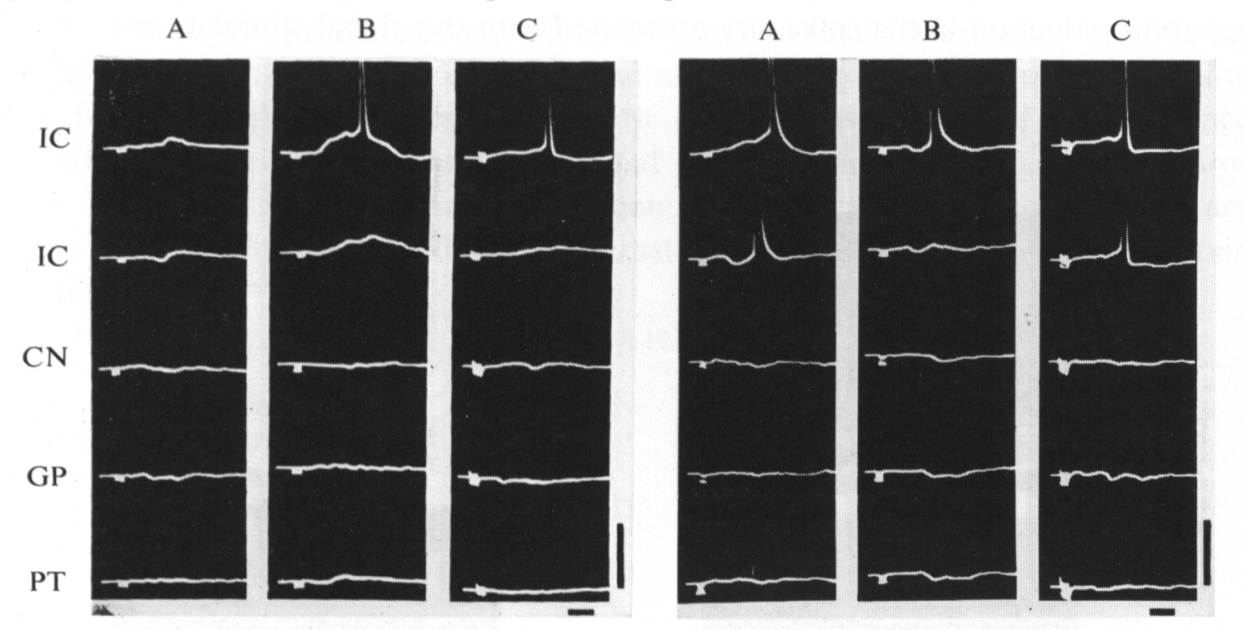

FIG. 3. (Left) Intracellular recordings from three flexor motoneurons responding to stimuli to contralateral internal capsule (IC), caudate nucleus (CN), globus pallidus (GP) and putamen (PT).

Two rows of IC illustrate records obtained when the polarity of stimulating currents was reversed between two electrodes, whose tips were $2 \mathrm{~mm}$. apart and both situated in the internal capsule. Time mark: 10 msec. Voltage calibration: $50 \mathrm{mV}$.

FIG. 4. (Right) Intracellular recordings from three extensor motoneurons. Description as in fig. 3.

In contrast to the midbrain stimulation described in the previous paper ${ }^{10}$ ) stimulation of the striate body was not always effective and, even in case effective, the result was less marked. The stimuli to the caudate nucleus, globus pallidus or putamen have never induced spike discharges either from flexor or from extensor motoneurons. On the contrary, IPSP's were predominant, which occurred with a latency of $10-15 \mathrm{msec}$. and continued for about $20 \mathrm{msec}$. as irregular deflections. In general, stimulation of the caudate nucleus was effective after a latency of a few milliseconds longer than that of the globus pallidus. This 
seems to agree with the generally accepted fiber connections within the striate body, that the caudate nucleus sends efferent fibers to the globus pallidus, which in turn sends efferent fibers to the lower brain stem either directly or indirectly ${ }^{*}$. The effect induced from the putamen was somewhat variable. Generally speaking, IPSP's were common events also in this case, but in a small number of motoneurons small EPSP's were observed instead of IPSP's.

The inhibitory action of the globus pallidus upon the monosynaptic reflex was In FIG. $5 \mathrm{~A}$, the upper beam illustrates a spike potential in a lumber motoneuron which was set up in response to a dorsal root stimulation. With a global stimulation antecedent by about $10 \mathrm{msec}$, the dorsal root stimulation induced only EPSP's without any spike discharge (FIG. $5 \mathrm{~B}$, upper beam). The lower beams in both records represent the ventral root outflows in these cases, which show an apparent reduction in the spike size associated with the global stimulation.

A

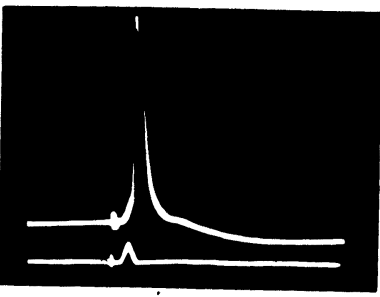

B
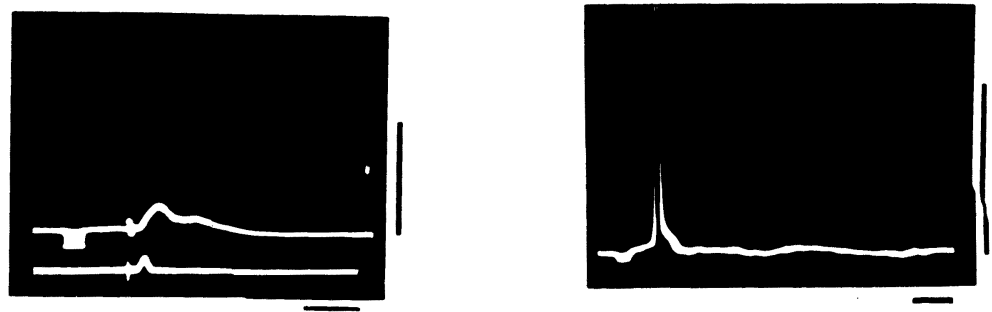

FIG. 5. (Left) Inhibition of monosynaptic reflex discharge from a spinal motoneuron effected by stimulating contralateral globus pallidus.

A : responses to a single dorsal root stimulation. Upper beam shows an intracellular recording from a lumbar motoneuron, lower beam monosynaptic reflex discharge recorded from the ventral roots.

B: responses when the same dorsal root stimulus was associated with a stimulus to globus pallidus. Upper beam shows abolition of the spike. Lower beam shows reduced spike discharge in the ventral roots.

Time mark: $10 \mathrm{msec}$. Voltage calibration : $50 \mathrm{mV}$ for upper beams.

FIG. 6. (Right) Intracellular recordings of spike and postsynaptic potentials from a extensor motoneuron induced by stimulating pyramidal decussation.

Time mark : $10 \mathrm{msec}$. Voltage calibration : $50 \mathrm{mV}$. 
The stimulation of the internal capsule set up EPSP's and in most cases a spike potential in a lumber alpha motoneuron. Not infrequently deflections which suggested the presence of IPSP's were observed on the records. Two stimulation foci which were $2 \mathrm{~mm}$. apart from each other produced in some cases similar results (FIG. 3 B, C; FIG. 4, B, C), in others qualitatively different results (FIG. 3 A; FIG. 4, A). These variable results characterized the effect of stimulation of the internal capsule, especially when it was compared with that of the pyramidal decussation. A stimulus to the pyramidal decussation always produced EPSP's and a consequent spike in a lumbar spinal motoneuron (FIG. 6). Apparently, loci in the internal capsule stimulated in the present experiment would have included fibers which belonged to the pyramidal as well as extrapyramidal system and therefore were manifold in their functions.

In FIG. 7 are presented four planes of Horsley-Clarke's Anterior 10, 12, 14 and 16 , in which the loci of stimulation are mapped with different marks indicating their functional characteristics. The most conspicuous feature of the influence exerted by the striate body upon the spinal motoneurons are the lack of reciprocality, in the sense that the influences upon flexor and extensor motoneurons are almost alike. This is in striking contrast to those exerted by the midbrain structures $^{10)}$.

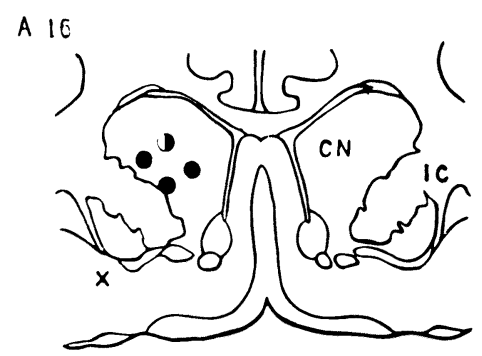

A 12

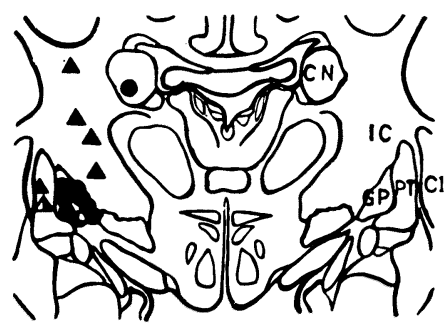

A 14

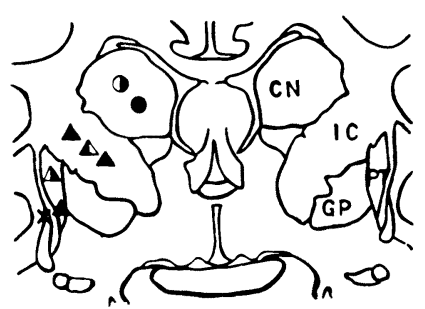

A 10

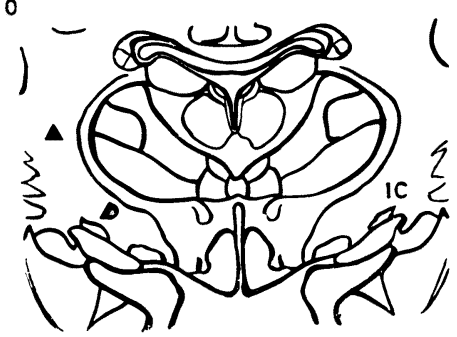

FIG. 7. Loci of stimulation mapped up in four planes of HorsleyClarke's Anterior 10, 12, 14 and 16.

Solid triangles: facilitatory points.

Solid circles: inhibitory points.

The left half of each symbol shows the effect on flexor motoneurons and the right half that on extensor motoneurons. Emptiness of one half of a symbol indicates ineffectiveness on either extensor or flexor motoneuron. Crosses indicate ineffectiveness on both of them. 


\section{(IV) Activities of the spinal interneurons.}

Sometimes the intracellular potentials were picked up from the spinal interneurons, when a microelectrode was pushed through the gray matter down to the motoneuron pool. Some of the interneurons responded to a dorsal root stimulation with several spikes in succession, whose initial one appeared with a latent time shorter than that for the motoneuronal spike. Summated EPSP's which appeared during and after the spike discharge characterized those responses of interneurons. The same interneurons were found to respond also to stimuli given to the striate body with such latencies, that some of them were slightly shorter than the average latency of motoneuronal response, while others were slightly longer than that. Some other interneurons responded to both dorsal root stimulation and a stimulus to the internal capsule. On the other hand, it is of interest, that interneurons which were responsive to the stimulation of the internal capsule or the pyramidal decussation were not responsive to the stimulation of the caudate nucleus and globus pallidus, and vice versa. Sometimes putamen proved to be an exception and excited an interneuron which responded to internal capsule stimulation. Full evaluation of this fact will need further researches.

A

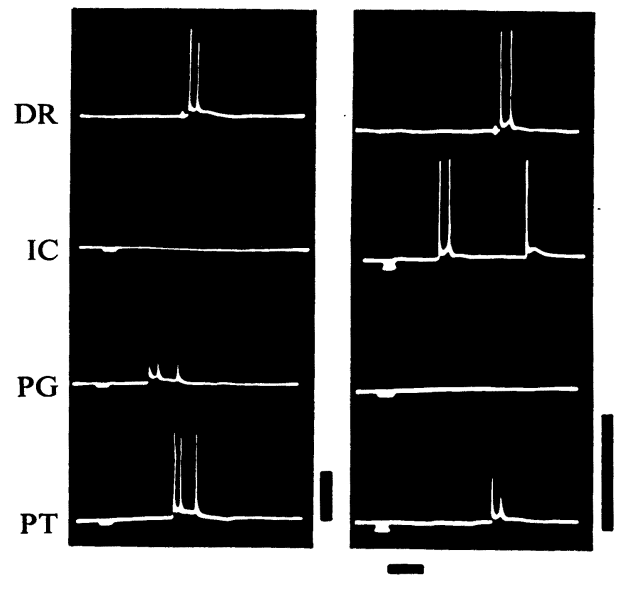

FIG. 8. Intracellular records from two interneurons which responded to dorsal root stimulation with short latency.

A : interneuron responded to striatal stimulation but not to internal capsule stimulation.

B : interneuron responded to stimuli given to internal capsule and putamen but not to a stimulus given to globus pallidus.

Time mark: $10 \mathrm{msec}$. Voltage calibrations : $50 \mathrm{mV}$.

\section{DISCUSSION}

There are a good many researches concerning inhibitory phenomena related to the caudate nucleus. Numerous stimulation experiments ${ }^{1,3,6,13)}$ as well as experiments involving ablation of the caudate nucleus, $\$, 11$ ) have suggested that the nucleus was inhibitory in function. On the other hand, researches dealing with movements elicited by stimulating caudate nucleus and others of the basal ganglia are also numerous. Among the reports of the latter group, Forman and WARD"' described recently movements elicited by stimulation of the caudate nucleus in unanesthetized cats. The movements were stereotyped and restricted to the con- 
tralateral side of the body. Also Segundo, Migliaro and Roig ${ }^{12)}$ described the effect of stimulating the striate complex in cats immobilized with Flaxedil. They found that the amplitude of the ventral roots response to dorsal root stimulation was either augmented by striatal (caudate, putamen, globus pallidus) and claustral excitation or, far more rarely, reduced (only from caudate and putamen). According to those authors, anesthetics in relatively small doses abolished this augmenting effect.

As stated above, the result of striate body stimulation in our present study was inhibitory to the spinal monosynaptic reflex, producing hyperpolarizing postsynaptic potentials in spinal motoneurons, with rare exceptions of facilitatory result in the case of putamen stimulation. The presence of these IPSP's was also endorsed by the positive electrotonic potential recorded from the ventral root. Hence, the present result is in agreement with earlier investigations ${ }^{1,3,6,13)}$ and in contradiction to more recent reports ${ }^{2}{ }^{12}$ ). At present, it seems hardly possible to reconcile these contradictory results. The usage of anesthetics would be an important factor in determining the result. However, though a moderate amount of Nembutal was used in our experiment, striatal stimulation was commenced always after the lapse of 5-7 hours with cats recovered almost completely from anesthesia. Intracellular recordings from the svinal motoneurons were accomplished during brief intervals of quiescence which intervened rather frequent, seemingly voluntary movements of limbs and trunk. Also the repetitive "spontaneous" firings of spinal motoneurons which were regularly found in the present research were in accordance with the presumed active state of the central nervous system $^{10)}$. It is therefore most unlikely that the usage of anesthetics caused to overlook the facilitatory function of the striate body which would have been discovered if otherwise. It seems more likely that the discrepant results should be attributed to other experimental conditions, such as strength and frequency of stimulation, mode of testing, etc.

Stimulation of the internal capsule and the pyramidal decussation served as a control to assure the above mentioned results of striatal stimulation as a real striatal function. As shown in Figs. 3 and 4, the result was quite different from that of striatal stimulation when the electrode tip was located in the internal capsule.

Intracellular recordings from the spinal motoneurons and interneurons have recently been performed by MORRELL ${ }^{7}$ ) associated with stimulation of medullary pyramid. Our results of pyramidal decussation stimulation seem to be mostly in agreement with his.

\section{SUMMARY}

1) The influence of stimulations of the striate body, internal capsule and pyramidal decussation upon the spinal activity was studied with cats under the slightest degree of Nembutal anesthesia. Intracellular potential recording from lumbar 
alpha motoneurons, as well as recording of ventral root electrotonic potentials, were performed in association with brain stimulations and sometimes also with dorsal root stimulations.

2) Stimulation of the caudate nucleus and globus pallidus reduced the size of spikes in the contralateral ventral roots discharged as a monosynaptic reflex by dorsal root stimulation. Striatal stimulation in isolation produced a positive ventral root potential.

3) Stimulation of the caudate nucleus and globus pallidus induced a train of irregular hyperpolarizing deflections in the membrane potential of the contralateral extensor and flexor motoneurons. These hyperpolarizing deflections, which are considered as IPSP's, abolished a spike discharge in a motoneuron reflexly induced by dorsal root stimulation. Similar, but somewhat less marked changes, with a few exceptional cases of facilitatory effect, were found when putamen was stimulated.

4) Stimulation of the internal capsule resulted in a facilitated monosynaptic discharge in the contralateral ventral roots. EPSP's and a consequent spike discharge were found by intracellular recording. Sometimes IPSP was recorded prior to EPSP.

5) EPSP and spike potential in spinal motoneurons were resulted from stimulation of the pyramidal decussation.

6) Some of the spinal interneurons responded both to dorsal root stimulation and to striated stimulation. Others were found responsive to dorsal root stimulation and stimulation of the internal capsule.

We are indebted to Prof. T. OTANI for advice and criticism.

\section{REFERENCES}

1) AUSTIN, G. AND JASPER, H. Diencephalic and striatal mechanisms of facilitation and inhibition. 81-83. XVIII int. physiol. Congr., Copenhagen, 1950.

2) Forman, D. AND WARD, J. W. Responses to electrical stimulation of caudate nucleus in cats in chronic experiments. J. Neurophysiol. 20:230-244. 1957.

3) Freeman, G. L. and Krasno, L. Inhibitory function of the corpus striatum. Arch. Neurol. Psychiat., Chicago. 44: 323-327. 1940.

4) Koizumi, K., Ushiyama, J. AND Brooks, C. M. A study of reticular formation action on spinal interneurons and motoneurons. Jap. J. Physiol. 9: 282-303. 1959.

5) Lindsley, D. B., Schreiner, L. H. AND Magoun, H. W. An electromyographic study of spasticity. J. Neurophysiol. 12: 197-205. 1949.

6) MEtTler, F. A. Pyramidal and extrapyramidal relationships. Res. Publ. Ass. nerv. ment. Dis. 21 : 150-227. 1942.

7) Morrell, R. M. Intracellular recording from spinal motoneurons following stimulation of medullary pyramids. Nature. 180:709. 1957.

8) Ranson, S. W. Anatomy of the nervous system. Philadelphia: W. B. Saunders Company, 1955. 
9) RICHTER, C. P. AND HINES, M. Increased spontaneous activity produced in monkeys by brain lesions. Brain 61:1-16. 1938.

10) Sasaki, K., Namikawa, A. and Hashiramoto, S. The effect of midbrain stimulation upon alpha motoneurons in lumbar spinal cord of the cat. Jap. J. Physiol. 10:303316. 1960.

11) Schreiner, L. H., Lindsley, D. B. And Magoun, H. W. Role of brain stem facilitatory systems in maintenance of spasticity. J. Neurophysiol. 12: 207-216. 1949.

12) Segundo, J. P., Migliaro, F. F. ANd Roig, J. A. Effect of striatal and claustral stimulation upon spinal reflex and strychnine activity. J. Neurophysiol. 21:391-399. 1958.

13) Tower, Sarah S. Extrapyramidal action from the cat's cerebral cortex: motor and inhibitory. Brain 59: 408-444. 1936. 